\title{
Indirect luminosity measurements with LHCb
}

\author{
T. Shears ${ }^{* \dagger}$ \\ University of Liverpool, UK \\ E-mail: tara@hep.ph.liv.ac.uk
}

\section{F. de Lorenzi, R. McNulty, D. Moran}

University College Dublin, Ireland

\begin{abstract}
We report on two studies of indirect luminosity measurements, using events containing muon final states, in LHCb. The first method exploits elastic two photon dimuon production in LHCb. The process has a theoretical uncertainty of less than $1 \%$, making it an ideal choice for use in performing an indirect luminosity measurement in hadron colliders. Strategies for triggering, selection and background rejection are discussed. Our studies indicate that a measurement precision of $1 \%$ can be obtained with $1 \mathrm{fb}^{-1}$ of data. The second method uses measurements of electroweak boson production where a similar precision can be obtained. It should be possible to measure the luminosity to better than $1 \%$ precision by combining both approaches.
\end{abstract}

European Physical Society Europhysics Conference on High Energy Physics

July 16-22, 2009

Krakow, Poland

\footnotetext{
*Speaker.

†n behalf of the LHCb collaboration
} 


\section{Introduction}

It is essential to have a precise measurement of integrated luminosity to measure absolute cross-sections and to estimate background contributions to new physics. The number of events, $N$, seen for a given process is related to the cross-section, $\sigma$, and the beam luminosity $L$ by $N=L / \sigma$.

Traditional luminosity measurements involve the direct measurement of the beam current and shape. However this technique has associated uncertainties of about $10 \%$. Indirect measurements of the luminosity can be obtained from applying $L=N / \sigma$ if one can find a process which is both theoretically well known and which produces a sufficient number of events that can be detected with a well-determined efficiency and purity.

In this short review two indirect luminosity measurements are discussed; the quantum electrodynamic (QED) process $p p \rightarrow p+\mu+\mu+p$; and $\mathrm{W}$ and $\mathrm{Z}$ boson production.

\section{Exclusive production of dimuons}

The QED process $p+p \rightarrow p+\mu+\mu+p$ is known to better than $1 \%$ theoretically, and has an effective cross-section of about $70 \mathrm{pb}$ inside the $\mathrm{LHCb}$ acceptance. It has a distinctive experimental signature of two back-to-back muons (in the transverse plane), in an otherwise empty event.

LHCb has a fast hardware trigger (L0) with an output rate of $1 \mathrm{MHz}$ and a software trigger (HLT), running on a computing cluster, with an output rate of $2 \mathrm{kHz}$. To select our signal, the trigger must record two types of event: single events, which contain a single collision of type $p p \rightarrow p+\mu+\mu+p$; and pile-up events, which contain more than 1 collision, one of which is $p p \rightarrow p+\mu+\mu+p$ and another which can be any type.

The L0 trigger requires at least one muon with transverse momenta $>1 \mathrm{GeV}$, in coincidence with a low number of hits in the SPD (Silicon Pad Detector). At HLT two muon candidates are required with an invariant mass exceeding $1 \mathrm{GeV}$. The trigger has an efficiency of $77 \%$ (64\%) for single (pile-up) events which contain two muons inside the LHCb acceptance.

Candidate events are selected offline [1] by requiring that no other tracks are consistent with the dimuon vertex, and that the dimuon transverse momentum is below $50 \mathrm{MeV}$. This selection has an efficiency of $17 \%$, and a purity of $96 \%$, where the dominant background arises from double pomeron exchange. With $100 \mathrm{pb}^{-1}$ of data the statistical precision of a luminosity measurement should be $3 \%$, reaching $1 \%$ with a dataset of $1 \mathrm{fb}^{-1}$.

\section{Vector boson production of muons}

Electroweak boson production is theoretically known to $2-3 \%$. Most of the uncertainty is due to knowledge of the parton distribution functions (PDFs), which have been calculated by several groups using global fits to experimental data.

LHCb has a high efficiency ( $>90 \%$ ) for triggering and reconstructing muons from $\mathrm{W}$ and $\mathrm{Z}$ bosons [2] inside the $\mathrm{LHCb}$ acceptance. In $100 \mathrm{pb}^{-1}$ of data about $20,000 \mathrm{Z}, 40,000 \mathrm{~W}^{-}$and 75000 $\mathrm{W}^{+}$will be selected. The luminosity extracted from these production measurements will thereore quickly become limited by the PDF uncertainties. 
To avoid this, an alternative analysis method has been devised [3]. The $\mathrm{W}^{+}, \mathrm{W}^{-}$and $\mathrm{Z}$ differential cross-sections in data, measured as a function of pseudorapidity, are fitted to theoretical prediction. A $\chi^{2}$ is formed between the number of observed and expected events in each pseudorapidity bin. The fit minimises the $\chi^{2}$ by allowing the shape and normalisation of the prediction to vary. The fitted normalisation corresponds to the integrated luminosity of the dataset, thus removing the need for an external measure. The fitted shape constrains the uncertainty in the parton density functions (the MSTW, CTEQ, Alekhin, NNPDF [4] models have been tested), whose uncertainty allows the shape to vary. These PDFs are constrained to vary within their current bounds. Only those theoretical predictions whose corresponding $\chi^{2}$ probability is greater than $1 \%$ are considered.

With $100 \mathrm{pb}^{-1}$ of data the luminosity is expected to be known up to $1.7 \%{ }^{1}$, decreasing to $1.3 \%$ with $1 \mathrm{fb}^{-1}$ and $0.8 \%$ with $10 \mathrm{fb}^{-1}$ of data. It should be noted that the goodness of fit implicit in the method will allow these different models to be distinguished in the data. Expected statistical errors for $10 \mathrm{fb}^{-1}$ of data, and different PDF models, are given in table 1 .

\begin{tabular}{|l|c|c|c|c|}
\hline & MSTW08 & CTEQ6.6 & Alekhin02 & NNPDF1.0 \\
\hline $\mathrm{W}^{+}$ & 1.3 & 2.0 & 1.5 & 2.1 \\
$\mathrm{~W}^{-}$ & 1.2 & 1.9 & 1.6 & 2.1 \\
$\mathrm{Z}$ & 1.4 & 1.9 & 1.9 & 1.8 \\
$\mathrm{~W}^{+}, \mathrm{W}^{-}, \mathrm{Z}$ & 0.8 & 1.7 & 1.0 & 1.3 \\
\hline
\end{tabular}

Table 1: Expected statistical precision on the luminosity with $10 \mathrm{fb}^{-1}$ of data, for fits to $\mathrm{W}$ and $\mathrm{Z}$ bosons separately and together, assuming four different parton density function models.

\section{Conclusions}

Two indirect luminosity measurement strategies are presented. The first, using a QED process, can reach a measurement precision of $1 \%$ with $1 \mathrm{fb}^{-1}$ of data. The second, using $\mathrm{W}$ and $\mathrm{Z}$ data, can reach a similar precision. Together, it should be possible to measure the luminosity to better than $1 \%$ precision.

\section{References}

[1] J. Anderson, "Testing the electroweak sector and determining the absolute luminosity at LHCb using dimuon final states", CERN-THESIS-2009-020.

[2] For a discussion of $\mathrm{W}$ and $\mathrm{Z}$ selections see T. Shears, these proceedings.

[3] F. de Lorenzi, "Indirect precise luminosity measurement with LHCb”, Deep Inelastic Scattering 2009.

[4] A. D. Martin et al., arXiv:hep-ex/0901.0002v2; P. Nadolsky et al. Phys. Rev. D78 013004 2008; S. Alekhin, Phys. Rev. D63 094022 2001; R. D. Ball et al. Nucl. Phys. B809 2009.

\footnotetext{
${ }^{1}$ depending on the PDF model employed
} 